\title{
Mamíferos do Parque Florestal Estadual do Rio Doce, Minas Gerais, Brasil.
}

\author{
Jody R. Stallings ${ }^{1}$ \\ Gustavo A. B. da Fonseca ${ }^{1}$ \\ Luiz Paulo de Souza Pinto ${ }^{1}$ \\ Ludmilla Moura de Souza Aguiar ${ }^{1}$ \\ Eduardo Lima Sábato ${ }^{2}$
}

\section{RESUMO}

Durante um periodo de 29 meses consecutivos foram realizados inventários de mamíferos em habitats de floresta nativa e exótica, além de área de campo, todos situados no Parque Florestal Estadual do Rio Doce, localizado na região ocidental da Mata Atlântica no estado de Minas Gerais. Foram registradas para o Parque um total de 60 espécies de mamíferos, distribuidas por 9 ordens, 24 famílias e 49 gêneros. De um esforço total de 64.300 armadilhas-noite, foram realizados 2.129 capturas de pequenos mamíferos não-voadores pertencentes a 20 espécies. Foram também capturadas 14 espécies de quirópteros, representando 12 gêneros e 4 famílias. Em $300 \mathrm{Km}$ de censos diumos repetidos nas áreas de mata nativa foram registradas 12 espécies de mamíferos de médio e grande porte, além de 14 outras observadas através de meios diversos.

Estes dados reforçam a idéia que a mastofauna da Mata Atlântica é bastante diversa. O Parque Estadual Florestal do Rio Doce deve ser considerado como uma das principais áreas para a conservação de fauna desta região e sua proteção deve ser priorizada.

\section{INTRODUÇÃO}

O estado de Minas Gerais possui cerca de $584.000 \mathrm{Km}^{2}$, compreendendo três tipos principais de biomas: Cerrado, Caatinga e Mata Atlântica. Dentre estes, a Mata Atlântica foi, por fatores históricos, aquele que sofreu o mais intenso processo de devastação, estando atualmente reduzido a aproximadamente $5 \%$ de sua cobertura vegetal original (Fonseca, 1985a). Provavelmente menos de $1 \%$ de sua extensão é representada por áreas não pertubadas (Mittermeier et alli, 1982).

Embora altamente ameaçada, a mastofauna da Mata Atlântica é pouco

1 Programa de Mestrado em Ecologia, Conservação e Manejo de Vida Silvestre, Instituto de Ciências Biologicas, Universidade Federal de Minas Gerais, Belo Horizonte, MG.

2. Departamento de Zoologia, Inctituto de Ciências Biológicas, Universidade Federal de Minas Gerais, Belo Horizonte, MG. 
conhecida. Os trabalhos pioneirọs nesta região indicam a existência de pelo menos 130 espécies de mamíferos não voadores (Fonseca \& Kierulff, 1989; Stallings, 1989), das quais 25 são endêmicas (Stallings \& Fonseca, em prep.). Destas, 15 estão incluídas na lista oficial das espécies ameaçadas de extinção do Instituto Brasileiro do Meio Ambiente e dos. Recursos Naturais Renováveis (Bernardes et alli, 1990). Quanto aos mamíferos voadores (Chiroptera), a lista mais recente indica a provável existência de 64 espécies para a região (Koopman, 1982), sendo que todas têm "status" de conservação desconhecido.

O Parque Florestal Estadual r's Rio Doce (PFERD), situado no Estado de Minas Gerais, foi objeto de t1 aoalhos que geraram listas qualitativas de mamíferos:(Gastal, 1982), de trabalhos quantitativos com pequenos mamíferos nậo voadores (Stallings, 1989; Fonseca \& Kierulff, 1989), de trabalhos sobre técnicas para a captura de pequenos mamíferos (Stallings et alli, 1987a, 1990a), de seleção de microhabitats (Stallings et alli, 1987b; 1987c; Fonseca \& Robinson, 1990), da variação temporal na captura de pequenos mamíferos (Stallings et alli, 1990b) e da importância de distúrbios na manutenção de diversidade de fauna (Stallings et alli, 1990c). Foram também realizados trabalhos com primatas (Santos et alli, 1983; Aguirre, 1971; Fonseca, 1985b; Mittermeier et alli, 1982; Stallings, 1988) e quirópteros (Bohoroquez \& Fonseca, 1983; Aguiar et alli, 1987; Stallings, 1988).

O objetivo deste trabalho é catalogar as espécies de mamíferos que ocorrem no PFERD e descrever os habitats onde foram observadas. Estes dados podem servir como subsídio para futuros trabalhos com mamíferos na área, e propiciar a comparaçã̀o com comunidades de mamíferos de outras regiōes Neotropicais.

\section{ÁREA DE ESTUDO}

O PFERD, administrado pelo Instituto Estadual de Florestas de Minas Gerais (IEF-MG), possui uma área aproximada de 35.000 hectares, estando localizado no vale do Rio Doce, face oeste da Serra da Mantiqueira (Municípios de Marliéria, Timóteo e Dionízio), entre as coordenadas de $19^{\circ} 48^{\prime}$ e $19^{\circ} 29^{\prime} \mathrm{S}$ e $42^{\circ} 38^{\prime}$ e $42^{\circ} 28^{\prime} \mathrm{O}$.

O clima é tropical úmido (Gilhuis, 1986), com chuvas entre os meses de novembro e fevereiro e uma estação seca de junho a agosto. A média anual de precipitaçāo, calculada para um período de vinte anos, foi de $1480 \mathrm{~mm}$ (CETEC, 1981).

A altitude do PFERD varia entre 230 e 515 metros. De acordo com o CETEC (1981), $21 \%$ do Parque é composto de planícies, $40 \%$ por morros e $34 \%$ por montanhas.

Além de ser a maior área preservada de Mata Atlântica do estado, o PFERD possui aproximadamente 40 lagoas e brejos resultantes da sedimentação dos lagos (Saijo \& Tundisi, 1985).

A vegetação do PFERD pode ser classificada como semi-decídua tropical 
(Gilhuis, 1986) e apresenta matas em diversos estágios de sucessão. Nos anos de 1964 e 1967 o Parque sofreu incêndios florestais que chegaram a consumir aproximadamente $30 \%$ de sua área (Stallings, 1988).

\section{MÉTODOS}

Este estudo foi realizado ao longo de um período de 29 meses, entre junho de 1985 e outubro de 1987, amostrando-se áreas distintas do PFERD. Pequenos mamíferos não voadores foram amostrados através do uso de três tipos de armadilhas (convencional com gancho, Tomahawk e Sherman), quirópteros com redes tipo mist-nest $(6 \times 2,5 \mathrm{~m})$ e mamíferos maiores através de censos diurnos e observações diretas no campo.

\section{1. Áreas Inventariadas.}

Sete áreas distintas foram levantadas, representando cinco tipos diferentes de habitat:

Mata primária - duas áreas amostradas (RD/T e RD/C). Estas áreas correspondiam, segundo classificação de Gilhuis (1986), a florestas primárias altas com epífitas.

Mata secundária heterogênea - duas áreas amostradas (RD/H e RD/M). Compreendem áreas alteradas por incêndios (1967) que afetaram a mata irregularmente, resultando em um mosaico de florestas de pequeno, médio e grande porte. Na classificação de Gilhuis (1986), corresponde a uma floresta de média para alta, com bambus e graminóides.

Mata secundária homogênea - uma área amostrada (RD/F). Esta área foi completamente queimada em 1967, apresentando atualmente uma mata baixa com sub-bosque bastante fechado e presença abundante de lianas. Corresponde a floresta secundária média com bambus e graminóides na classificação de Gilhuis (1986).

Campo sujo associado com brejo - uma área amostrada (RD/B), sendo intermediária entre terrenos alagados e secos. Na classificação de Gilhuis (1986), corresponde a dois tipos vegetacionais: arvoredo baixo e savana altigraminosa sazonal com arbustos e árvores baixas.

Floresta de eucalipto com espécies nativas - área homogênea de eucalipto com sub-bosque bem desenvolvido, formado por espécies nativas. Uma área amostrada (RD/E), que se localizava fora dos limites do Parque.

A duração do trabalho nas sete áreas não foi a mesma, tendo sido efetuados 29 meses de amostragem nas áreas RD/H e RD/C, 22 meses em RD/M e RD/F, 18 meses em RD/B e 12 meses em RD/T e RD/E. 


\section{Métodos de amostragem}

\section{Pequenos mamíferos}

Em cada área de mata amostrada foram estabelecidas três trilhas paralelas, distanciadas entre si por 100 metros. Cada trilha era composta de 16 postos de captura equidistantes 20 metros, perfazendo um total de 300 metros por trilha. As armadilhas, em um total de 112 por área, foram dispostas no chão e a uma altura média de um a dois metros em todos postos. Stallings (1989) e Fonseca \& Kierulff (1989) fornecem maiores informaçōes sobre a metodologia. Nas áreas compostas de matas mais altas (RD/C, RD/T, RD/H e RD/M) foram também colocadas armadilhas nas copas das árvores, através de plataformas (Stallings et alii, 1987a e 1990a). Estas foram colocadas a uma altura média de 10 metros, perfazendo, nestas quatro áreas, um total de 42 armadilhas. Na área de campo sujo (RD/B) foram estabelecidas duas trilhas de 280 metros cada, distanciadas 50 metros. Cada trilha era composta de 20 postos, cada um com 2 armadilhas (todas no chão), totalizando 80 armadilhas.

O estudo com pequenos mamíferos não-voadores constou de um programa de captura-marcação-recaptura, e para cada indivíduo capturado eram anotadas as seguintes informaçōes: espécie, localização (área, trilha, posto), identificação individual (presença de anilha), medidas morfométricas, peso, sexo, idade e condicão reprodutiva. Os indivíduos foram identificados através de uma coleção de referência, além de consulta a coleções e o envio de espécimes para a identificação por especialistas. A coleção de referência está depositada na Universidade Federal de Minas Gerais.

\section{Mamiferos de Médio e Grande Porte.}

Para outros mamíferos não voadores foram feitos censos durante o período de novembro de 1985 a outubro de 1986, nas seguintes áreas: RD/C, RD/T, $\mathrm{RD} / \mathrm{H}, \mathrm{RD} / \mathrm{M}$ e RD/F. Estes censos foram feitos, para cada área amostrada, nas trilhas de captura de pequenos mamíferos. Para cada indivíduo observado eram anotadas (a) distância em metros da trilha, (b) altura em que se encontravam (no caso de espécies arbóreas), (c) hora do dia, (d) tamanho do grupo e (e) outros dados biológicos. As trilhas eram percorridas a uma velocidade aproximada de $1 \mathrm{Km}$ por hora, correspondendo a uma distância de 1051 metros por área amostrada por dia. Isto representou cerca de $60 \mathrm{Km}$ de censos repetidos por cada área amostrada durante o período de um ano.

\section{Quirópteros}

Morcegos foram coletados de modo sistemático e não sistemático em vários tipos de habitat (mata primária, mata secundária, campo sujo) e também em ambientes com influência antrópica, como residências, ancoradouros, ruínas e estradas. As redes de captura eram abertas pouco antes de escurecer e permaneciam assim por cerca de três horas. "Para cada indivíduo capturado 
eram anotadas a espécie, a hora de captura, medidas morfométricas, peso, sexo, idade e condicâo reprodutiva. Alguns indivíduos foram anilhados. Foi feita também uma coleção de referência que está depositada na Universidade Federal de Minas Gerais.

\section{Terminologia Adotada.}

Para pequenos mamíferos, a primeira captura é definida como a primeira vez em que um indivíduo é capturado e anilhado. $O$ total de capturas inclui as recapturas subsequentes.

A abundância relativa é determinada pela razão entre o número de indivíduos capturados ( $1^{\mathrm{a}}$ captura $)$ de uma determinada espécie e o número total de primeiras capturas de todas as espécies.

O esforço de captura ou número de armadilhas-noite foi calculado multiplicando-se o número de armadilhas pelo número de noites em que elas permaneceram abertas em cada área por mês.

Para quirópteros, a abundância relativa foi calculada pela razão entre o número de indivíduos capturados de cada espécie e o número total de indivíduos capturados de todas as espécies.

Para mamíferos maiores, que foram objeto de censos nos transectos, a abundância relativa foi calculada usando a média de indivíduos observados por quilômetro.

\section{RESULTADOS}

\section{Composiçāo da fauna de mamíferos do PFERD}

Durante o período de junho de 1985 a outubro de 1987, considerando amostragens sistemáticas e nāo sistemáticas, foram registradas um total de 60 espécies de mamíferos para o PFERD. As espécies estão distribuídas entre 9 ordens, 24 famílias e 49 gêneros (Tabela 1). As espécies da ordem Rodentia e Chiroptera representaram $28,3 \%$ e $23,3 \%$, respectivamente, de todas as espécies nativas observadas. As outras 7 ordens contribuiram com $48,3 \%$ das espécies. Em termos de adaptação espacial das taxa, $52,2 \%$ das espécies são terrestres, $26,1 \%$ escansoriais e $21,7 \%$ arborícolas.

\section{Roedores, Marsupiais e Quirópteros}

Ao longo de 29 meses de amostragem sistemática de pequenos mamíferos não-voadores, com um esforço total de 64.300 armadilhas-noite, foram realizadas 2.129 capturas de 1.067 indivíduos, pertencentes a 20 espécies, sendo 8 marsupiais e 12 roedores (Tabela 2 ). 
Tabela 1. Espécies de mamíferos registradas para o Parque Florestal Estadual do Rio Doce, dispostas em ordem taxônomica, com abundância relativa, tipo de registro e adaptação espacial (segundo Stallings, 1989 e Fonseca \& Kierulff, 1989). Abundância relativa: $\mathrm{A}=$ muito comum; $\mathrm{B}=$ comum; $\mathrm{C}=$ incomum; e $\mathrm{D}=$ raro. Tipo de registro: 1 = armadilha, $2=$ censo; $3=$ observação oportunística. Adaptação Espacial: $\mathrm{A}=$ arborícola; $\mathrm{T}=$ terrestre; $\mathrm{E}=$ escansorial.

\begin{tabular}{llll}
\hline Espécie & Abundância & $\begin{array}{l}\text { Tipo de } \\
\text { Registro }\end{array}$ & $\begin{array}{l}\text { Adaptação } \\
\text { Espacial }\end{array}$ \\
\hline
\end{tabular}

\section{MARSUPIALIA}

Didelphidae

Marmosa cinerea

$M$. incana

A

A

Gracilinanus sp

Monodelphis americana

Monodelphis sp

Metachirus nudicaudatus

Didelphis marsupialis

Caluromys philander

C

C

C

A

A

B

$\begin{array}{ll}1 & \text { A } \\ 1 & \text { E } \\ 1 & \text { E } \\ 1 & \text { T } \\ 1 & \text { T } \\ 1 & \text { E } \\ 1,3 & \text { A }\end{array}$

\section{RODENTIA}

\section{Cricetidae}

Oryzomys capito

O. subflavus

$\begin{array}{lll}\text { A } & 1 & T\end{array}$

O. trinitatis

B

O. nigripes

Abrawayaomys ruschii

Néctomys squamipes

Rhipidomys mastacalis

Akodon cursor

A

Oxymycterus roberti

Calomys laucha

Muridae

Rattus rattus

D

Echimyidae

Euryzygomatomys guiara

Caviidae

Cavia sp

A

C

1

T

1,3
A

E

E

$T$

$T$

E

A 
Vol. 7(4). 1990

Tabela 1. Continuação.

\begin{tabular}{llll}
\hline Espécie & Abundância & $\begin{array}{l}\text { Tipo de } \\
\text { Registro }\end{array}$ & $\begin{array}{l}\text { Adaptaçāo } \\
\text { Espacial }\end{array}$ \\
\hline
\end{tabular}

Sciuridae

Sciurus aestuans

B $1,2,3 \quad$ A

Hydrochaeridae

Hydrochaeris hydrochaeris

A

3

T

Dasyproctidae

Dasyprocta azarae

Agouti paca

$\begin{array}{lll}\text { A } & 1,2,3 & \text { T } \\ \text { B } & 1,3 & \text { T }\end{array}$

\section{CHIROPTERA}

Phyllostomatidae

Anoura caudifer

B 1

A. geoffroyi

$\begin{array}{ll}\text { C } & 1\end{array}$

Artibeus lituratus

A 1

A. jamaiscenses

B 1

Carollia perspicillata

A 1

Chrotopterus auritus

$\begin{array}{ll}\text { C } & 1\end{array}$

Glossophaga soricina

B 1

Micronycteris $\mathrm{sp}$

C 1

Phyllostomus hastatus

C 1

Sturnira lilium

Vampyrops lineatus

$\begin{array}{ll}\text { A } & 1\end{array}$

Vespertilionidae

Myotis sp

A

1

Noctilionidae

Noctilio leporinus

A

1

Emballonuridae

Rhynchonycteris naso

C 1

Cebidae

Alouatta fusca

C 2

C 2

Brachyteles arachnoides

A

A

2,3

A

A

Cebus apella

Callicebus personatus

2,3

A

A

\section{EDENTATA}

Myrmecophagidae

Tamandua tetradactyla

C 
Tabela 1. Continuação

\begin{tabular}{l}
\hline Espécie \\
\hline Bradypodidae
\end{tabular}

Bradypus variegatus

C

3

A

Dasypodidae

Euphractuş sexcinctus

Dasypus novemcinctus

C

2,3

A

3

$T$

T

\section{CARNIVORA}

\section{Canidae}

Dusicyon thous

$\begin{array}{lll}\text { A } & 3 & T \\ \text { C } & 3 & \text { E } \\ \text { C } & 2,3 & \text { E }\end{array}$

Nasua nasua

A

3

E

Eira barbara

Felidae

Felis pardalis

B

F. concolor

B

3

B

F. yagouaroundi

C

3

E

3

F. onca

3
$B$

$T$

3

E

T

\section{PERISSODACTYLA}

\section{Tapiridae}

Tapirus terrestris

A

2,3

T

ARTIODACTYLA

Cervidae

Mazama sp

C

2,3

T

\section{LAGOMORPHA}

Leporidae

Sylvilagus brasiliensis

A

3

T

Analisando as capturas por tipo de habitat, verifica-se que em áreas de mata nativa, com um esforço de captura de 55.060 armadilhas-noite, roedores apresentaram maior riqueza de espécies, enquanto marsupiais apresentaram. 
Vol. 7(4), 1990

Tabela 2. Número de indivíduos (ind) e capturas (cap) totais de pequenos mamíferos não-voadores em diferentes tipos de habitat no Parque Floresta Estadual do Rio Doce. $\mathrm{A}=$ mata nativa; $\mathrm{B}=$ campo sujo $\mathrm{C}=$ área de eucalipto com sub-bosque nativo na borda do Parque.

\begin{tabular}{|c|c|c|c|c|c|c|c|c|}
\hline \multirow[t]{2}{*}{ Espécie } & \multicolumn{2}{|c|}{$\mathbf{A}$} & \multicolumn{2}{|c|}{ B } & \multicolumn{2}{|c|}{ C } & \multicolumn{2}{|c|}{ Total } \\
\hline & ind & cap & ind & cap & ind & cap & ind & cap \\
\hline Marmosa cinerea & 178 & 523 & 2 & 2 & 9 & 75 & 189 & 600 \\
\hline M. incana & 126 & 206 & 3 & 4 & 8 & 14 & 137 & 224 \\
\hline Gracilinanus sp. & 1 & 1 & 0 & 0 & 0 & 0 & 1 & 1 \\
\hline $\begin{array}{l}\text { Monodelphis } \\
\text { americana }\end{array}$ & 1 & 1 & 0 & 0 & 0 & 0 & 1 & 1 \\
\hline Monodelphis sp. & 2 & 2 & 0 & 0 & 0 & 0 & 2 & 2 \\
\hline $\begin{array}{l}\text { Metachirus } \\
\text { nudicaudatus }\end{array}$ & 138 & 203 & 1 & 2 & 9 & 17 & 148 & 222 \\
\hline $\begin{array}{l}\text { Didelphis } \\
\text { marsupialis }\end{array}$ & 51 & 66 & 1 & 3 & 3 & 7 & 55 & 76 \\
\hline Caluromys philander & 58 & 101 & 1 & 2 & 6 & 16 & 65 & 119 \\
\hline Total & 555 & 1103 & 8 & 13 & 35 & 129 & 598 & 1245 \\
\hline
\end{tabular}

\section{ROEDORES}

\begin{tabular}{|c|c|c|c|c|c|c|c|c|}
\hline Oryzomys capito & 74 & 95 & 5 & 11 & 2 & 2 & 81 & 108 \\
\hline O. subflavus & 9 & 20 & 24 & 47 & 0 & 0 & 33 & 67 \\
\hline O. trinitatis & 55 & 73 & 5 & 13 & 0 & 0 & 60 & 86 \\
\hline O. nigripes & 0 & 0 & 10 & 10 & 0 & 0 & 10 & 10 \\
\hline $\begin{array}{l}\text { Abrawayaomys } \\
\text { ruschii }\end{array}$ & 1 & 1 & 0 & 0 & 0 & 0 & 1 & 1 \\
\hline $\begin{array}{l}\text { Nectomys } \\
\text { squamipes }\end{array}$ & 12 & 21 & 11 & 14 & 0 & 0 & 23 & 35 \\
\hline $\begin{array}{l}\text { Rhipidomys } \\
\text { mastacalis }\end{array}$ & 4 & 11 & 0 & 0 & 0 & 0 & 4 & 11 \\
\hline Akodon cursor & 75 & 132 & 146 & 389 & 16 & 25 & 237 & 546 \\
\hline Oxymycterus roberti & 4 & 3 & 5 & 6 & 0 & 0 & 9 & 9 \\
\hline Rattus novergicus & 1 & 1 & 0 & 0 & 0 & 0 & 1 & 1 \\
\hline Calomys laucha & 0 & 0 & 8 & 8 & 0 & 0 & 8 & 8 \\
\hline
\end{tabular}

\section{MARSUPIAIS}

maior abundância relativa, perfazendo mais de $70 \%$ dos indivíduos. Este padrão nâo se repete na área de campo sujo, com um esforço de captura de 3.240 
armadilhas-noite, onde roedores foram mais abundantes, sendo responsáveis por $96 \%$ dos indivíduos. $\mathrm{Na}$ área de eucalipto, com um esforço de capturâ de 6.000 armadilhas-noite, marsupiais foram mais abundantes e estão representados por um número maior de espécies do que os roedores.

Das 20 espécies de pequenos mamíferos não voadores, somente sete (Marnosa cinerea, M. incana, Metachinus nudicaudatus, D. marsupialis, C. philander, $O$. capito e A. cursor) estão representados em todos os ambientes amostrados. As espécies mais abundantes, considerando os três tipos de habitat, foram $A$. cursor e $M$. cinerea, sendo responsáveis respectivamente, por 22 e $18 \%$ dos indivíduos capturados.

Com um esforço de captura de 84 horas-rede e capturas ocasionais, foram realizadas 103 capturas de quirópteros pertencentes a 14 espécies, representando 12 gêneros e 4 famílias (Tabela 3). Indivíduos anilhados nunca foram recapturados. Somente as espécies $C$. perspicillata e $A$. lituratus estão representadas em todos os ambientes amostrados. Estas são também as espécies

mais abundantes, contribuindo com $65 \%$ das capturas. A família Phyllostomatidae representou $78,7 \%$ das espécies e $94 \%$ das capturas das 4 famílias amostradas.

\section{Outros Mamíferos}

Em $300 \mathrm{Km}$ de censos diurnos repetidos nas áreas de mata nativa, foram registradas 11 espécies de mamíferos, além de um caso de hibridismo, possivelmente entre $C$. penicillata, introduzido no PFERD (Coimbra-Filho, 1984), e a espécie nativa do Parque, C. aurita (Tabela 4).

Outras 13 espécies de mamíferos registradas para o Parque foram observadas através de capturas ocasionais com armadilhas em diversas áreas, observações oportunísticas e incursões noturnas de carro ao longo da estrada principal do Parque.

\section{DISCUSSĀO}

Este estudo propiciou a determinacão da riqueza e da abundância relativa de mamíferos de uma área de floresta Neotropical através de coleta, censos sistemáticos e de documentação visual.

A análise dos padrões de ocorrência de espécies nos distintos tipos de habitat revelou uma distribuição diferencial para alguns grupos. No caso de primatas, um grupo de hábitos predominantemente arbóreos, houve uma tendência de ocorrência de um maior número de espécies nos habitats de mosaicos $(\mathrm{RD} / \mathrm{H} \mathrm{e}$ $\mathrm{RD} / \mathrm{M})$, quando comparados com matas primárias ( $\mathrm{RD} / \mathrm{C}$ e $\mathrm{RD} / \mathrm{T}$ ) e a mata secundária homogênea $(\mathrm{RD} / \mathrm{F})$. Para outras espécies de médio e grande porte, não houve uma preferência por este tipo de habitat. Em geral, a área de mata 
secundária homogênea apresentou um número menor de espécies do que as matas primárias, e as matas heterogêneas.

Com respeito a pequenos mamíferos, $A$. cursor dominou a comunidade na área de campo (RD/B), sendo responsável por $67 \%$ e $78 \%$ dos indivíduos capturados e das capturas totais, respectivamente. Estes dados reforçam as observações de Fleming (1975) e sugerem que uma ou duas espécies usualmente dominam uma dada comunidade de pequenos mamíferos. Nas áreas de mata não houve uma dominância evidente como ocorreu na área de campo. Os marsupiais $M$. cinerea, $M$. nudicaudatus e $M$. incana representavam $22 \%, 17 \%$ e $16 \%$ dos indivíduos capturados $(36 \%, 14 \%$ e $14 \%$ das capturas totais), respectivamente. Cinco outras espécies de roedores e marsupiais foram responsáveis por entre $6 \%$ e $9 \%$ do número total de capturas, com mais 8 espécies dos dois grupos representando menos de $1 \%$ dos indivíduos capturados. Neste caso, a comunidade de pequenos mamíferos não parece ser dominada por um pequeno número de espécies.

Certamente existem outras espécies de mamíferos no PFERD que não foram catalogadas durante nosso estudo. $\mathrm{O}$ baixo esforço de captura para quirópteros

Tabela 3. Espécies e número de capturas de quirópteros em diferentes tipos de habitat no Parque Florestal Estadual do Rio Doce. $\mathrm{A}=$ mata nativa; $\mathrm{B}=$ ambientes antrópicos; $\mathrm{C}=$ campo sujo.

\begin{tabular}{lllll}
\hline Espécie & A & B & C & Total \\
\hline Anoura caudifer & 0 & 5 & 0 & 5 \\
A. geoffroyi & 0 & 1 & 0 & 1 \\
Artibeus literatus & 5 & 21 & 1 & 27 \\
A. jamaiscensis & 1 & 0 & 0 & 1 \\
Carollia perspicillata & 15 & 24 & 1 & 40 \\
Chrotopterus auritus & 1 & 0 & 0 & 1 \\
Glossophaga soricina & 0 & 9 & 0 & 9 \\
Micronycteris sp & 1 & 1 & 0 & 2 \\
Phyllostomus hastatus & 3 & 0 & 0 & 3 \\
Sturnira lilium & 4 & 0 & 0 & 4 \\
Vampyrops lineatus & 0 & 3 & 1 & 4 \\
Rhynchonyteris naso & 0 & 1 & 0 & 1 \\
Myotis sp & 0 & 1 & 0 & 1 \\
Noctilio leporinus & 0 & 4 & 0 & 4 \\
No de capturas & & & & 103 \\
\hline
\end{tabular}


Tabela 4. Espécies observadas e média de indivíduos por quilômetro, registrados por censos de transecto, em cinco áreas de mata no Parque Florestal Estadual do Rio Doce.

\begin{tabular}{llllll}
\hline & \multicolumn{5}{c}{$\mathbf{N}^{\mathbf{i}} \mathbf{i n d} / \mathbf{k m}$} \\
Espécie & $\mathbf{R D} / \mathbf{F}$ & $\mathbf{R D} / \mathbf{H}$ & $\mathbf{R D} / \mathbf{M}$ & $\mathbf{R D} / \mathbf{T}$ & $\mathbf{R D} / \mathbf{C}$ \\
\hline Callicebus personatus & 0,17 & 0,20 & 0,44 & - & - \\
Cebus apella & - & 0,58 & 0,27 & 0,30 & 0,40 \\
Callithrix aurita & - & - & 0,02 & 0,08 & 0,03 \\
Callithrix sp (híbrido) & - & 0,47 & - & - & - \\
Alouatta fusca & - & 0,05 & - & - & - \\
Brachyteles arachnoides & - & - & 0,30 & - & - \\
Sciurus aestuans & 0,02 & 0,27 & 0,05 & - & 0,02 \\
Dasyprocta azarae & - & 0,10 & 0,05 & 0,13 & 0,20 \\
Nasua nasua & 0,02 & - & 0,02 & 0,07 & - \\
Tapirus terrestris & 0,02 & 0,02 & - & 0,02 & - \\
Mazama sp & - & - & - & - & 0,20 \\
Euphractus sexcinctus - & - & - & - & 0,02 & - \\
\hline
\end{tabular}

explica o pequeno número de espécies amostradas. Segundo Koopman (1982), 58 espécies de quirópteros têm uma distribuição potencial na área do PFERD. Isto significa que o esforço foi suficiente para o registro de, no mínimo, $24 \%$ da riqueza existente. Não foram feitos censos noturnos para a documentação de espécies de mamíferos não voadores e, além disso, problemas como o tipo de isca, tipo de armadilha utilizada, comportamento de espécies, etc., ajudam a explicar porque vários grupos são sub-representados pelos nossos métodos. Com base nos mapas disponíveis de distribuiçōes potenciais de mamíferos Neotropicais (vide Mares \& Genoways, 1982), espera-se que outras 22 espécies de mamíferos possam ocorrer no PFERD, não constando pois na lista apresentada neste estudo. Estes dados elevam para 68 o número de espécies de mamíferos não-voadores com ocorrência potencial no PFERD, sendo que nossas obervações representaram portanto $67,6 \%$ deste total. Apesar dos problemas citados acima; a relação de espécies de mamíferos registrada para o PFERD é representativa quando comparada com outras listas de mamíferos obtidas em diferentes áreas de planícies úmidas da América do Sul. A comparação com listas de espécies para áreas pontuais da Amazônia (Cosha Cashu, no Parque Nacional de Manu, no Peru, e do Projeto Áreas Mínimas nas proximidades de Manaus, Brasil; Emmons, 1984) e da Mata Atlântica Argentina (Parque Nacional Iguaçú; Crespo, 1982), indica que a fauna de PFERD é relativamente rica (Figura 1). Estes dados evidenciam uma tendência de que as áreas Amazônicas, especialmente as de Cosha Cashu, possuem uma maior riqueza de espécies de primatas e de roedores, em relação à Mata Atlântica. 


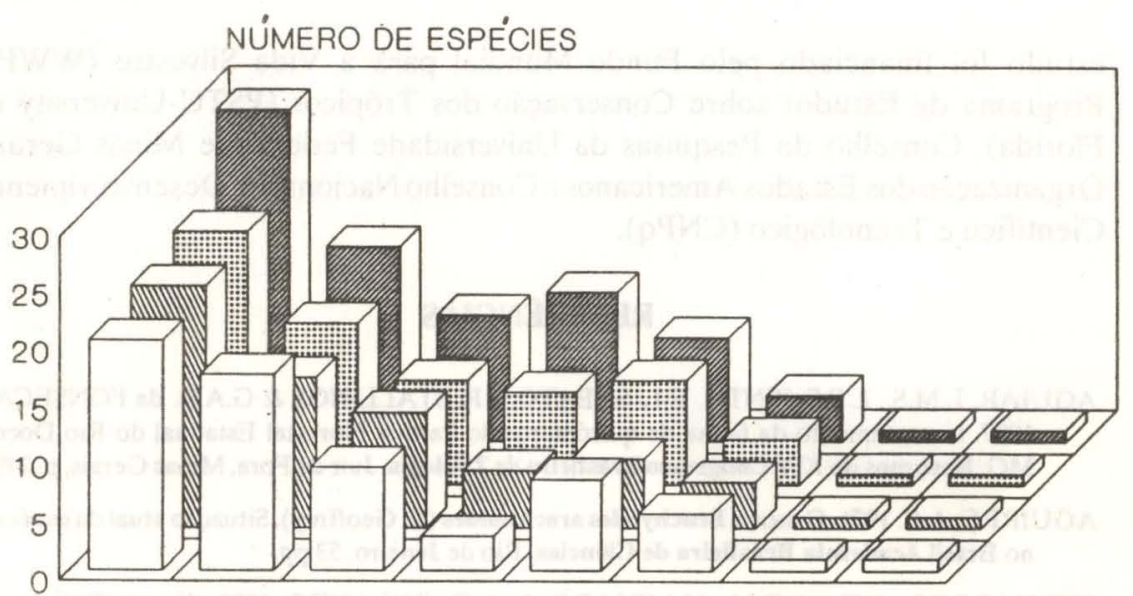

RODEN. CARNI. MARSU. PRIMA. EDENT. ARTIO. LAGOM. PERIS.

ORDENS

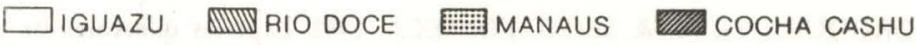

FONTES: EMMONS, 1984; CRESPO, 1982

Figura 1. Riqueza de espécies de mamíferos em quatro áreas da América do Sul: Parque Nacional Iguazu (Crespo, 1982); Cosha Cashu no Perú e uma área próxima à Manaus, Brasil (Emmons, 1984); e Parque Florestal Estadual do Rio Doce, Minas Gerais, Brasil.

Esta diferença pode ser explicada, entre outras razões, pela manutenção da relação entre número de espécies e o tamanho da área comparada (MacArthur e Wilson, 1967). Para os outros grupos, a biodiversidade observada é similar entre as diferentes regiões.

Embora ainda possua uma mastofauna rica em espécies, o Parque constitui-se em um dos últimos refúgios representativos da biodiversidade da Mata Atlântica do estado de Minas Gerais, mostrando a importância de sua proteção e manejo adequados.

\section{AGRADECIMENTOS}

Gostaríamos de agradecer à P. Procópio e L. C. Bedê pela ajuda na coleta dos dados de campo. Também ao Instituto Estadual de Florestas de Minas Gerais que proporcinou acomodacão no Parque e, em especial, à administração do Parque, nas pessoas de A. Lopes, H. Silva-Neto e J. Ladeira, que ajudaram nos problemas logísticos diários. Gostaríamos de agradecer também a M. Carleton, K. Creighton, L. Emmons, G. Musser, P. Myers e J. Patton, que generosamente identificaram alguns espécimens de pequenos mamíferos. Este 
estudo foi financiado pelo Fundo Mundial para a Vida Silvestre (WWF), Programa de Estudos sobre Conservação dos Trópicos (PSTC-University of Florida), Conselho de Pesquisas da Universidade Federal de Minas Gerais, Organização dos Estados Americanos e Conselho Nacional de Desenvolvimento Científico e Tecnológico (CNPq).

\section{REFERENCIAS}

AGUIAR, L.M.S., L.P.S. PINTO, E.L. SÁBATO, J.R. STALLINGS \& G.A.B. da FONSECA. 1987. Levantamento da fauna de quirópteros do Parque Florestal Estadual do Rio Doce, MG. Resumos do XIV Congresso Brasileiro de Zoologia. Juiz de Fora, Minas Gerais, p. 249:

AGUIRRE, A.C. 1971. O mono Brachyteles arachnoides (E. Geoffroy). Situação atual da espécie no Brasil.Academia Brasileira de Ciências, Rio de Janeiro. 53 pp.

BERNARDES, A.T., A.B.M. MACHADO \& A.B. RYLANDS. 1990. Fauna Brasileira Ameaçada de Extinção. Fundação Biodiversitas para a Conservaçāo da Diversidade Biológica. Belo Horizonte. MG. 62 pp.

BOHOROQUEZ, G. M. \& M.C.G. FONSECA. 1983. Espécies diferentes de quirópteros Hemiderma perspicillatum Trouessant, 1904, e Lonchoglossa ecaudata Lima, 1926 (Mammalia: Chiroptera), co-habitando em uma mesma colónia. Resumos do X Congresso Brasileiro de Zoologia, Belo Horizonte, Minas Gerais. pp:386-387.

CETEC. 1981. Vegetação do Parque Florestal do Rio Doce. Programa de Pesquisas Ecológicas no Parque Florestal Estadual do Rio Doce. Relatório Final. Vol.2. Fundação Centro Tecnológico de Minas Gerais, Belo Horizonte, Minas Gerais, 277 pp.

COIMBRA-FILHO, A.F. 1984. Situação atual dos calitriquideos que ocorrem no Brașil. p. 15-33. In: M.T. de Mello (ed.), A Primatologia no Brasil. Sociedade Brasileira de Primatologia, Brasilia, D.F.

CRESPO, J.A. 1982. Ecologia de la comunidad de mamiferos del Parque Nacional Iguazu, Misiones. Revista Mus. Argentino Cien. Nat. "Bernadino Rivadavia". Instituto Nacional de Investigación de la Ciencias Naturales, 3(2):45-162.

EMMONS, L.H. 1984. Geographic variation in densities and diversities of non-flying mammals in Amazonia. Biotropica, 16(3):210-222.

FLEMING, T.H. 1975. The role of small mammals in tropical ecosystems. pp. 269-298. In: F.B. Golley, K. Petrusewicz \& L. Ryszkowski (eds.), Small Mammals: their productivity and populations dynamics. International Biological Program, Cambridge University Press.

FONSECA, G.A.B. 1985a. The vanishing Brazilian Atlantic Forest. Biological Conservation. 34(1):17-34.

FONSECA, G.A.B. 1985b. Observations on the ecology of the muriqui (Brachyteles arachnoides E. Geoffroy 1806): implications foi its conservation. Primate Conservation, 5:48-52

FONSECA, G.A.B. \& M.C.M. KIERULFF, 1989. Biology and natural history of Brazilian Atlantic Forest small mammals. Bulletin Florida State Museum, 34(3): 99-152.

FONSECA. G.A.B. \& J.G. ROBINSON. 1990. Forest size and structure: competitive and predatory effects on small mammal communities. Biological Conservation, 53:265-294.

GASTAL, M.L.A. 1982. Nota prévia sobre os marsupiais do Parque Estadual do Rio Doce, Minas Gerais Resumos do IX Congresso Brasileiro de Zoologia, Brasilia, D.F. p. 30-31. 
GILHUIS, J.P. 1986. Vegetation survey of the Parque Florestal Estadual do Rio Doce, MG, Brazil. Tese de Mestrado, Agricultural University of Wageningen, Netherlands. Viçosa, Universidade Federal de Viçosa/Agricultural University of Wageningen. 86 pp.

KOOPMAN, K.F. 1982. Biogeography of the bats of South America. p. 273-302. In: M.A. Mares \& H. H. Genoways (eds.) Mammalian Biology in South America. Special Publication Series. Vol.6. University of Pittsburgh. Linesville, Pittsburgh.

MACARTHUR, R. \& E. WILSON. 1967. The theory of Island Biogeography. Princenton University Press, Princeton. 203 pp.

MARES, M.A. \& H.H. GENOWAYS. 1982. Mammalian Biology in South America. Special Publication Series. Pymatuning Laboratory of Ecology. Vol.6. University of Pittsburgh. Linesville, Pittsburgh. 539 pp.

MITTERMEIER, R.A., A.F. COIMBRA-FILHO, I.D. CONSTABLE, A.B. RYLANDS \& C.M. VALLE.1982. Conservation of primates in the Atlantic Forest of Brazil. Int. Zoo. Yearbook 22:2-17.

SAIJO, Y. \& J.G. TUNDISI. 1985. Limmological studies in central Brazil. Rio Doce Valley lakes and pantanal wetland. $1^{\text {st }}$. report. Laboratory of Chemical Biology, Water Research Institute. Nayoga University, Chikusa-ku Nayoga, Japan. 201 pp.

SANTOS, 1: B., C.M.C. VALLE, M.C. ALVES \& R. A. MITTERMEIER. 1983. Levantamento preliminar da fauna de primatas do Parque Florestal Estadual do Rio Doce, (PFERD), Timóteo, MG, Brasil. Resumos do X Congresso Brasileiro de Zoologia. Belo Horizonte, Minas Gerais. p.397-398.

STALLINGS, J.R. 1988. Small mammal comunities in an eastern Brazilian park. Tese de Doutorado, University of Florida, Gainesville, Florida, EUA. 199 pp.

STALLINGS, J.R. 1989. Small mammal inventories in an eastern Brazilian park.Bulletin Florida State Museum 34(4): 153-200.

STALLINGS, J.R., L.M.S. AGUIAR, E.L. SÁBATO \& L.P.S. PINTO. 1987a. Captura de pequenos mamíferos nos estratos superiores de matas do Parque Florestal Estadual do Rio Doce, MG. Resumos do XIV Congresso Brasileiro de Zoologia. Juiz de Fora, Minas Gerais. p. 181.

STALLINGS, J.R., L.P.S. PINTO, E.L. SÁBATO \& L.M.S. AGUIAR. 1987b. Levantamento de pequenos mamíferos não-voadores em um área de transição entre brejo e mata no Parque Florestal Estadual do Rio Doce, MG. Resumos do XIV Congresso Brasileiro de Zoologia. Juiz de Fora, MG. p.181.

STALLINGS, J.R. , E.L. SÁBATO, L.M.S. AGUIAR, L.P.S. PINTO \& G.A.B. FONSECA. 1987c. Levantamento de pequenos mamíferos não-voadores em áreas florestais do Parque Florestal Estadual do Rio Doce, MG. Resumos do XIV Congresso Brasileiro de Zoologia. Juiz de Fora. MG. p.182.

STALLINGS, J.R., E.L. SÁBATO, M.C.M. KIERULFF, L.P.S. PINTO \& P.P. OLIVEIRA. 1990a. A importância do uso de plataformas na amostragem sistemática de pequenos mamíferos nos estratos superiores de matas primária e secundária na Mata Atlântica. Resumos do XVII Congresso Brasileiro de Zoologia. Londrina, PR. p.223.

STALLINGS, J.R., E.L. SÁBATO, P.P. OLIVEIRA, L.P.S. PINTO \& L.M.S. AGUIAR. 1990b. A variação na sazonalidade temporal da micro mastofauna na Mata Atlântica. Resumos do XVII Congresso Brasileiro de Zoologia. Londrina, PR. p.215.

STALLINGS, J.R., L.P.S. PINTO, L.M.S. AGUIAR \& E.L. SÁBATO. 1990c. A importância dos distúrbios intermediários na manutenção da diversidade da fauna em uma floresta tropical. p. 43-58. In: R.P. Martins \& F.S. Lopes (eds.), Atlas do Encontro de Ecologia Evolutiva. Academia de Ciências de São Paulo, Brasil. Publicação ACIESP. nº 69. 